\title{
Three-Dimensional Structure of Drosophila Testis Tip: The Spatial Relation Between Dividing Cells
}

\author{
Kyung Eun Lee, ${ }^{1, \star}$ Sung Sik Han, ${ }^{2}$ and Hyesung Jeon ${ }^{3}$ \\ ${ }^{1}$ Advanced Analysis Center, Korea Institute of Science and Technology, Hwarangno 14-gil 5, Seongbuk-g, \\ Seoul 136-791, Republic of Korea \\ ${ }^{2}$ School of Life Sciences and Biotechnology, Anam-dong, Seongbuk-gu, Seoul 136-701, Republic of Korea \\ ${ }^{3}$ Center for Theragnosis, Korea Institute of Science and Technology, Hwarangno 14-gil 5, Seongbuk-g, Seoul 136-791, \\ Republic of Korea
}

\begin{abstract}
At the apical tip of Drosophila testis, there is a stem cell niche known as the proliferation center, where the stem cells are maintained by hub cell cluster for the regulation of differentiation and proliferation. Germline stem cells go through mitosis four times from one primary spermatogonial cell to the 16-cell stage before the maturation. The cells derived from the same germline stem cell are located within one cyst, an enclosed system by two cyst cells, and they are connected by the intercellular bridges called ring canals. In this study, the three-dimensional (3D) structure of Drosophila testis tip was reconstructed from serial sections. The size of cells at each stage was compared in volume from the 3D structure. The stages of cells in a cyst could be distinguishable exactly by counting the cells linked with intercellular bridges in 3D-reconstructed structure. The cysts containing the same stage cells appeared in the horizontal plane. Both the germline stem cell directly attached to the hub cell and the spermatogonial cells detached from the hub cell were divided at the almost perpendicular direction to the spermatogonial cell layers. The dividing phase in one cyst was delayed gradually through the cytoplasmic region of intercellular bridge.
\end{abstract}

Key words: 3D reconstruction, Drosophila, testis, stem cell niche, spermatogenesis, cell division

\section{INTRODUCTION}

The adult Drosophila testes are a pair of coiled tubes in the abdomen. At the apical tip of the testis, cells are divided and differentiated to spermatid. At the end of the tip, there is a stem cell niche known as proliferation center, where the stem cell is maintained and the differentiation and proliferation is regulated (Hardy et al., 1979). Several small somatic cells are gathered at the center of the tip and form a tight clump known as a hub cell cluster. The germline stem cells (GSCs), which have a large nucleus and undifferentiated organelles in small cytoplasm, are attached to the hub cell cluster (Hardy et al., 1979). This contact between GSC and hub cell is the key factor for maintaining stem cell properties that GSC becomes GSC, not to be differentiated (Xie \& Spradling, 2000). During spermatogenesis, asymmetric division occurrs at the apical end of the tip. The attached cell to hub cells becomes a stem cell and the detached cell from hub cells is differentiated to primary spermatogonial cell (Yamashita et al., 2005).

The primary spermatogonial cell is enclosed in two cyst cells and they comprise a cyst, the fundamental unit of the spermatogenesis (Fig. 1). A primary spermatogonial cell passes through four mitosis, spermatocyte growth, meiosis, and spermatid differentiation in synchrony within the given cyst, and the cohort of germline cells within one cyst originates from the same primary spermatogonium. After four phases of mitosis division, a primary spermatogonial cell becomes 16 spermatogonia called as 16-cell stage. Pre-

() MICROSCOPY SOCIETY OF AMERICA 2013

${ }^{\star}$ Corresponding author. E-mail: kelee@kist.re.kr meiotic DNA replication occurs and then follows cell growth and gene expression in this 16-cell stage. Spermatogonial cells are different from primary spermatocytes, in that they possess many mitochondria and enlarged cytoplasm (Fuller, 1993).

During each cell division process before spermatid differentiation, intercellular bridges are formed by incomplete cytokinesis between newly dividing cells. In other words, primary spermatogonial cell is divided into two cells linked by one intercellular bridge within a given cyst, and thus this is referred to as the two-cell stage. The next mitosis makes four-cell stage linked by three intercellular bridges, and sequentially eight-cell stage with seven bridges and 16-cell stage with 15 bridges (Robinson \& Cooley, 1996). The intracellular bridge called the ring canal is a characteristic

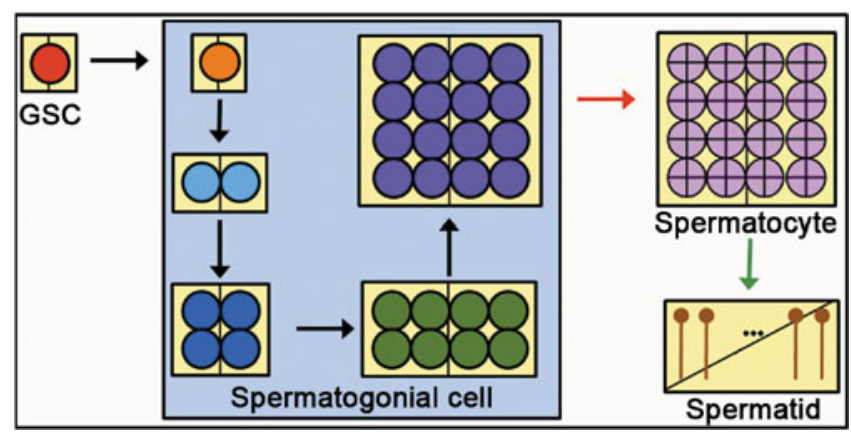

Figure 1. Spermatogenesis of Drosophila. Black arrow shows mitosis, red arrow shows meiosis, and green arrow shows maturation. Yellow represents the two cyst cells in a cyst. GSC, germline stem cell. 

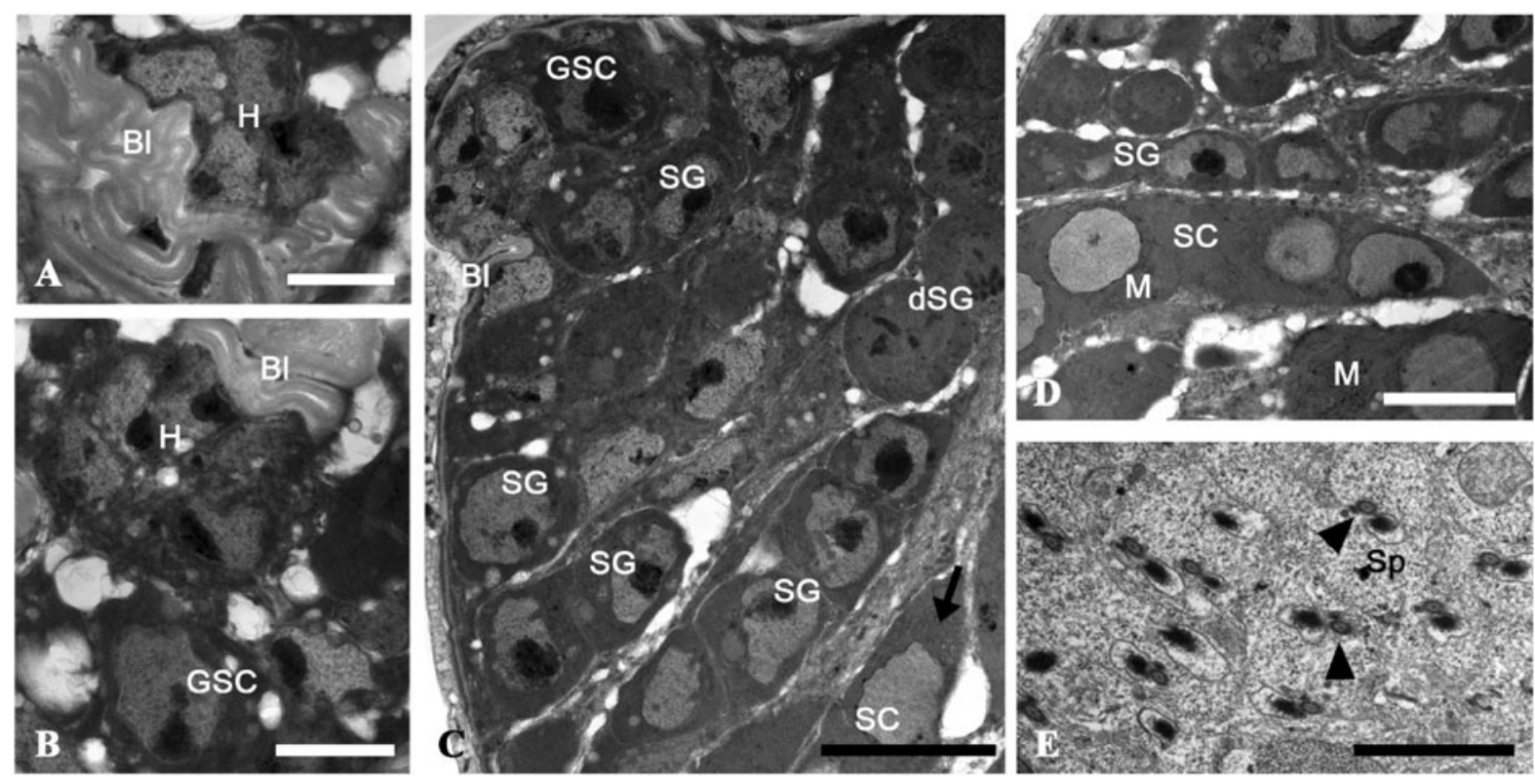

Figure 2. TEM micrographs of testis tip: (A, B) cross view; (C) longitudinal view; (D) 16-cell stage after premeiotic S phase. Each cell was further differentiated and showed enlarged cytoplasm and many mitochondria. (E) Matured spermatid. Arrow indicated the enlarged cytoplasm and arrowheads indicated the axoneme. Bl, basal laminar; dSG, dividing spermatogonial cell; GSC, germline stem cell; H, hub cell; M, mitochondria; SC, spermatocyte; SG, spermatogonial cell; Sp, spermatid. Scale bar: (A, B) $4 \mu \mathrm{m},(C, D) 10 \mu \mathrm{m}$, and (E) $3 \mu \mathrm{m}$.

feature of germline cell differentiation. This ring canal is derived from the contractile ring, which divides the cytoplasm for cell division, and germ cells within a given cyst may communicate with each other through this intercellular bridge (Hime et al., 1996). There is a large cytoplasmic structure called the fusome in the cytoplasmic region of the ring canal (Lin et al., 1994). After meiosis, 64 spermatids are still linked with the intercellular bridge. During the maturation process, intercellular bridges disappear and sperm cells are separated.

In this paper, the three-dimensional (3D) structure of Drosophila testis tip was reconstructed. From these results, we could confirm the exact division stage with cell numbers linked by intercellular bridge and know the sequence of cells in the mitosis part of spermatogenesis.

\section{Materials and Methods}

\section{Cultivation of Drosophila}

Drosophila melanogaster (Oregon-R standard strain) were raised on corn meal-yeast agar medium at $25^{\circ} \mathrm{C}$. Corn mealyeast agar contained $9 \mathrm{~g}$ of corn meal, $5 \mathrm{~g}$ of sucrose, $1.5 \mathrm{~g}$ of yeast extract, and $1 \mathrm{~g}$ of agar in $100-\mathrm{mL}$ distilled water.

\section{Transmission Electron Microscopy (TEM) Preparation}

Testes from 2-day-old adult Drosophila were dissected in modified insect buffer (Beller et al., 2006). They were fixed with $2.5 \%(\mathrm{v} / \mathrm{v})$ glutaraldehyde in $\mathrm{PBS}$ for $3 \mathrm{~h}$ at $4^{\circ} \mathrm{C}$. After washing with $0.1 \mathrm{M}$ cacodylate buffer three times, the specimens were post fixed in $2 \%$ osmium tetroxide solution for $3 \mathrm{~h}$ at $4^{\circ} \mathrm{C}$. Then they were washed and stained en bloc with $2 \%$ uranyl acetate in distilled water at $4^{\circ} \mathrm{C}$ overnight. The specimens were dehydrated in an ethanol series $(50,70$, $80,90,95$, and $100 \%$ ) for 15 min each and embedded in Spurr's resin (Spurr, 1969). The embedded specimens were sectioned serially at a thickness of $500 \mathrm{~nm}$ on MT-XL ultramicrotome (RMC, USA) using a diamond knife, and the serial sections were collected on the formvar-coated slot-grid. It was stained with $2 \%$ uranyl acetate in $50 \%$ methanol followed by lead citrate, and coated with carbon evaporator (Polaron, Quorum technology, England). The grids were observed using Tecnai F20 (FEI, the Netherlands).

\section{D Reconstruction}

One section image of the testis was acquired from stitching several partial TEM images of one section by PTgui, photo stitching software. The $3 \mathrm{D}$ model of the testis tip was reconstructed with IMOD image processing package (Kremer et al., 1996). Serial images were first aligned manually and then processed with cross-correlation. The serial images were aligned using the Midas program and contoured using $3 \mathrm{dmod}$. The $3 \mathrm{D}$ models were visualized using the IMODV program.

\section{Results AND Discussion}

A hub cell cluster surrounded with thick basal lamina was shown in the center of the apical end of testis tip (Fig. 2A) and GSCs were attached at the hub cells (Fig. 2B). Below the hub cell cluster, the serial layers of sequentially differentiated cells appeared (Fig. 2C). GSCs were adhering to the hub cell and the primary spermatogonial cells were laid on a layer below the GSCs (Fig. 2C). Dividing spermatogonial 

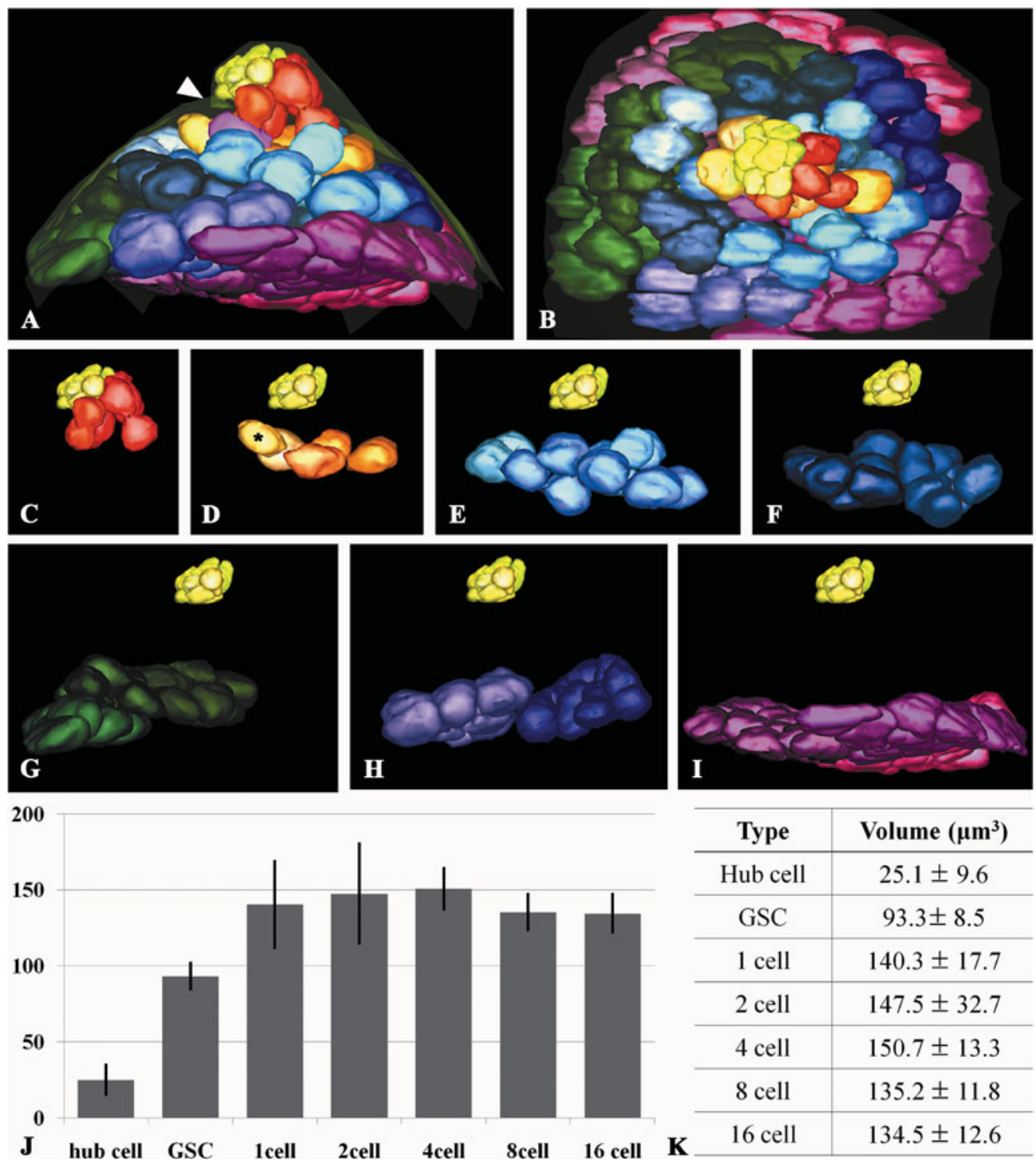

\begin{tabular}{c|c}
\hline Type & Volume $\left(\boldsymbol{\mu m}^{3}\right)$ \\
\hline Hub cell & $25.1 \pm 9.6$ \\
\hline GSC & $93.3 \pm 8.5$ \\
\hline 1 cell & $140.3 \pm 17.7$ \\
\hline 2 cell & $147.5 \pm 32.7$ \\
\hline 4 cell & $150.7 \pm 13.3$ \\
\hline 8 cell & $135.2 \pm 11.8$ \\
\hline 16 cell & $134.5 \pm 12.6$ \\
\hline
\end{tabular}

Figure 3. Three-dimensional (3D) reconstructions of Drosophila testis tip. The overall structure reconstructed from serial sections at side view (A) and top view (B). The same stage cells were displayed with hub cell cluster in (C-I). Gonial stem cells (C); one-cell stage primary spermatogonial cells (D), two-cell stage spermatogonial cells (E), four-cell stage cells $(\mathbf{F})$, eight-cell stage cells $(\mathbf{G})$, dividing eight-cell stage to 16-cell stage (H), 16-cell stage cells (I). (J, K) The volume of cells. Yellow, hub cell cluster (apical cells); red, germline stem cell (GSC); orange, primary spermatogonial cell; sky blue, two-cell stage spematogonial cell (two cells connected by intercellular bridge); blue, four-cell stage spermatogonia; dark green, eight-cell stage spermatogonia; purple, dividing spermatogonia; pink, 16-cell stage spermatogonia.

cells were also observed at the longitudinal view (Fig. 2C). The 16-cell staged primary spermatocyte, which was previous to meiosis, had the enlarged cytoplasm and the sharply increased mitochondria. The fully differentiated spermatid containing axoneme structure appeared at the location far from the test tip. In these 2D TEM images, all stages of spermatogonial cells before premeiotic $S$ phase showed similar structures, containing a large nucleus, undifferentiated organelles, and a small cytoplasm. Therefore, each stage of the spermatogonial cell was indistinguishable by its cellular structure itself. However, the exact stage of the spermatogonial cell can be discriminable by the cell counting linked with the intercellular bridge. In order to confirm the exact differentiated stage, 3D structure had to be reconstructed.
Cells in the apical tip were displayed well by 3D reconstructed structure (Fig. 3). The same stage cells were gathered composing a layer. GSCs were attached at the hub cell directly (Fig. 3C). Basal lamina spread into the inside of the apical region and it discontinued the contact of hub cell with GSC (Fig. 3A, arrowhead). One-cell stage spermatogonial cells were laid in the one layer (Fig. 3D). The two-cell stage spermatogonia, which went through first mitotic division and were connected by one intercellular bridge, formed a layer under primary spermatogonia (Fig. 3E). The directions of two-cell stage spermatogonia were near horizontal to cell layer plane (Fig. 3E). The clusters of four-cell stage cells after secondary mitotic division were also assembled into a layer (Fig. 3F). Spermatogonia of eight-cell and 

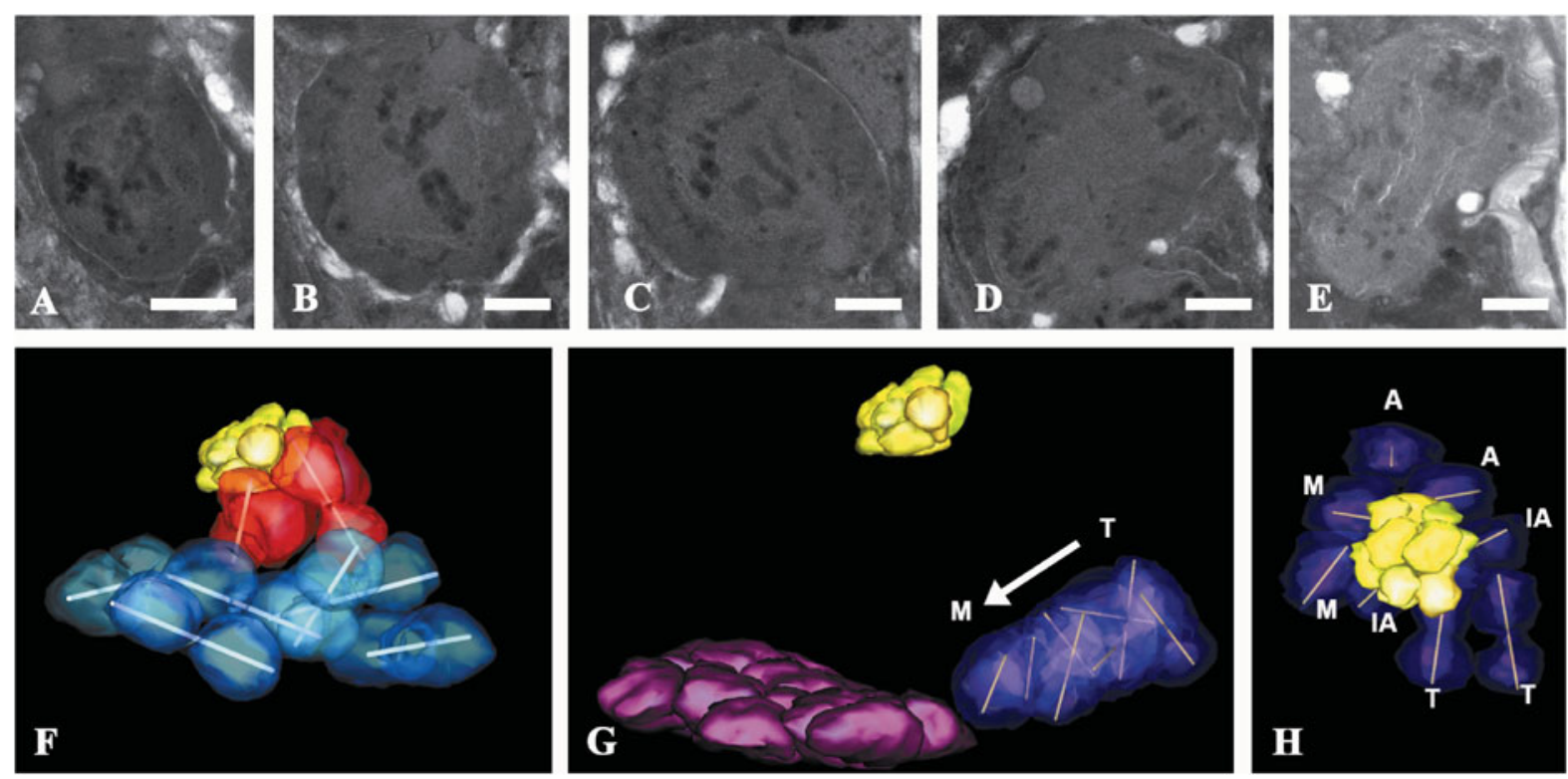

Figure 4. Three-dimensional structure of dividing cells: (A) prophase cell, (B) metaphase cell, (C) early anaphase cell, (D) late anaphase cell, (E) telophase and early cytokinesis cell. F: Hub cell cluster, germline stem cells, and two-cell stage spermatogonia. Two-cell stage cells were displayed with sky blue color and the directions of cells were displayed by white stick. G: Side view of the dividing eight-cell stage and 16-cell stage cells. Yellow sticks display the dividing directions. Arrow indicates the order of mitotic phase. H: Top view of the dividing eight-cell stage. A, anaphase; $\mathrm{M}$, metaphase; $1 \mathrm{~A}$, late anaphase; T, telophase. Scale bar: (A-E) $2 \mu \mathrm{m}$.

16-cell stage were also shown to build up a layer each other at the horizontal plane (Figs. 3G, 3I). From 3D reconstructed structure, the volume of each cell was calculated (Figs. 3J, 3K). The spermatogonial cells had similar volume in each cell stage, although they were about 1.5 times larger than GSCs and five to six times larger than hub cells. The marked cell in Figure 3D is the first cell under the hub cell cluster, but the direct contact on hub was interrupted by basal laminal canyon. The volume of this cell was about $132.1 \mu \mathrm{m}^{3}$, much larger than the average volume of GSC. This result implies that the direct contact to the hub cell should be kept to maintain the properties of GSC, which have a large nucleus and undifferentiated organelles in a small cytoplasm.

The dividing cells were clearly observed in the TEM images of testis tip (Fig. 4). In the prophase, the chromosomes were condensed (Fig. 4A). In the metaphase, the chromosomes started to align in a line at the center of cell (Fig. 4B). The sister chromatids began to move to the opposite end of the dividing cell in the early anaphase (Fig. 4C), and they appeared at the near opposite end of cell in the late anaphase (Fig. 4D). In the telophase, cytokinesis occurred, and thus the center of the cell was caved (Fig. 4E). GSC was divided at the perpendicular direction to the hub cell cluster (Fig. 4F). Even though the dividing spermatogonial cells far from the hub cell looked randomly divided at the top view (Fig. 4G), they were divided perpendicularly at the side view like the division of GSC attached at hub cluster (Fig. 4H).

In the $3 \mathrm{D}$ reconstructed structure, the hierarchy of divided cells could be guessed by counting intercellular bridges. The numbers of cells in Figure 5 were given by the manner of the fusome polarity model, which explains that the fusome had grown having polarity in oogenesis (Lin \& Spradling, 1995). In the four-cell stage, newly divided daughter cells have one intercellular bridge, whereas original mother cells have two bridges. In the TEM images (Fig. 5A), the inside region of the ring canal and some spreading region to connecting cytoplasm had shown low electron density and contained fusome, and had a highly branched vesicular structure (Lighthouse et al., 2008). The region containing fusome of four-cell stage was displayed in the center of the four-cell stage cluster (Fig. 5B). It was suggested that fusome was an important feature of Drosophila germline cell division regulation (Deng \& Lin, 1997; Kloc et al., 2004). The branched vesicular structure was a part of a single continuous endoplasmic reticulum (ER), which was shared by all dividing ovarian cells. The fusomal ER mediated intercellular ER connectivity by linking the cytoplasmic ER membrane, and thus fusome membrane and luminal proteins were diffused freely and rapidly between the connected cells. This ER continuity was suggested to have a possible role in synchronizing mitotic cyst divisions (Snapp et al., 2004).

Mitosis occurred at the perpendicular direction to the same staged cell layer. After completion of mitosis, however, cells were distributed at a horizontal plane by a layer. This phenomenon may be achieved by relocation of the cells (Fig. 5B, arrows). The newly formed fusomes derived from incomplete cytokinesis move to fuse together toward original fusome after finishing one cycle of mitosis, and enlarged fusome branches are formed at the center of a cohort of cells (de Cuevas \& Spradling, 1998). When mitosis started, 

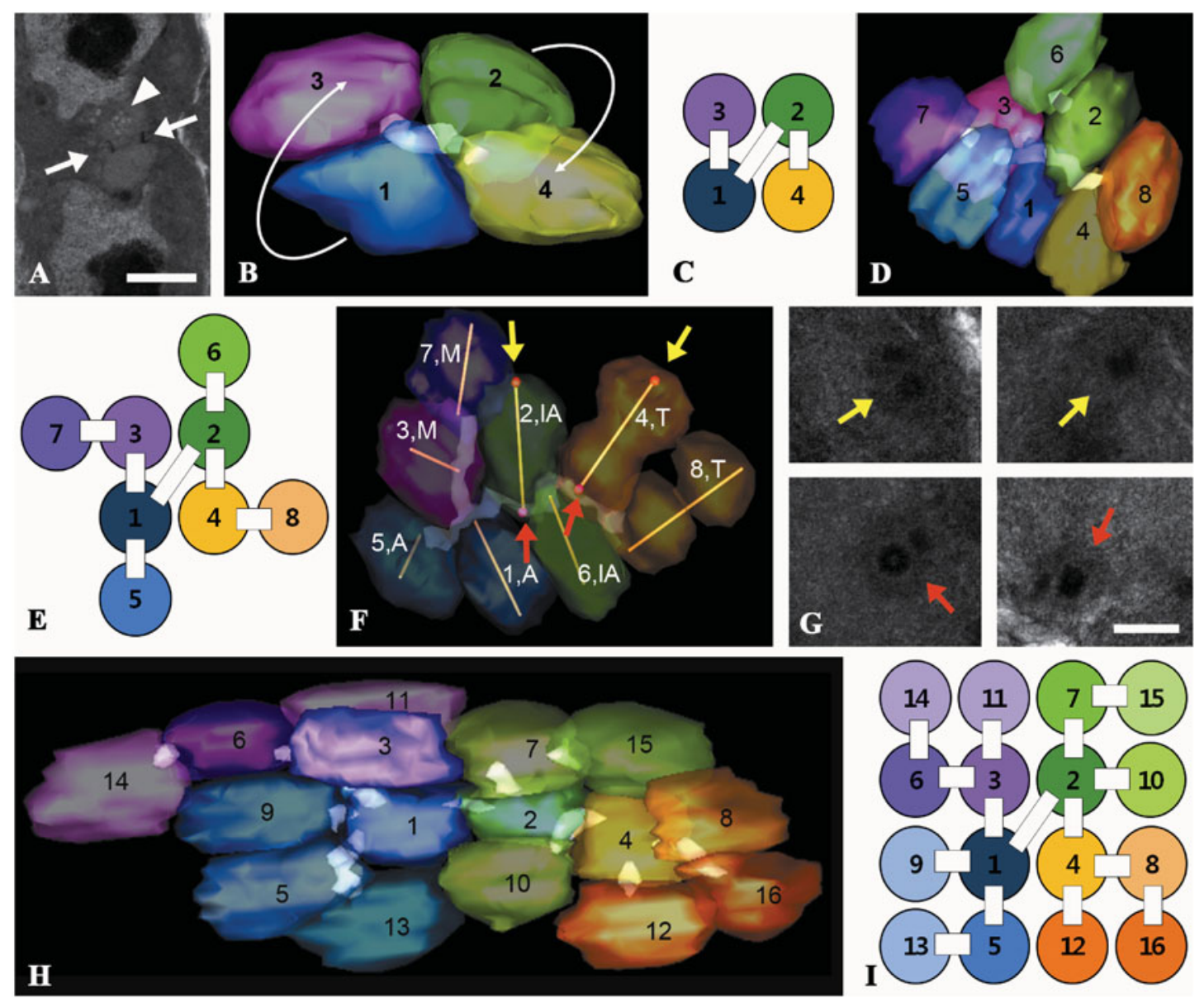

Figure 5. Intercellular bridges and cell numbering by their linkages. A: Transmission electron microscopy (TEM) image of the region containing fusome. Highly branched vesicular structure was indicated by arrowhead; arrows indicate a ring canal, and low electron density cytoplasm inside of ring canal was marked with asterisk. B: Top view of the four-cell stage. Arrows indicate the guessed direction of the ring canal moving. C, E, I: The brief diagrams of numbered spermatogonial cells and intercellular bridges, (D) eight-cell stage, (F) dividing eight-cell stage. Yellow sticks indicate the direction of division. G: TEM images of indicated region by arrows in (F). Red arrows indicate the centrosomes in the intercellular bridge. Yellow arrows indicate the counterpart centrosomes. H: 16-cell stage cells. Scale bar: (A) $2 \mu \mathrm{m}$ and (G) $600 \mathrm{~nm}$

the mother centrosomes appeared at the inside region of the ring canal, the center region of the cell cluster, and daughter centrosomes appeared at the counter part (Fig. 5G). Ring canals originating from contract rings were located in the center between mother and daughter centrosome laid on the perpendicular direction during mitosis (Fig. 5F, displayed by yellow stick). When cell division is complete, the fusome gathered the center of the cyst, and the ring canals were also relocated to the center (Deng \& Lin, 1997). This process may be the force for relocating the daughter cells at a horizontal layer.

It was suggested that the mitotic synchrony was regulated through the fusome (Snapp et al., 2004). Figure 5F showed a little different time phase of the cell division. The division phase was delayed gradually through the region containing fusome. The division phase was started from the cell marked with number 8 which was located at the end of fusome, rather than from the centered cells marked with number 1 or 2 which was the original mother cell (Figs. 5E,
5F). The polarity was toward the original cell during oogenesis in female Drosophila. Thus, it is determined as the future oocyte and future nurse cells (Lin \& Spradling, 1995). In Figure 5F, the polarity of division signaling appeared through the region containing fusome, but it was not converged on spermatocyte 1 . During oogenesis, only one cell inside a cyst becomes oocyte, and the residual cells become supporting cells as the future nurse cells. Compared with this asymmetric cell division in female oogenesis, spermatogonial cells are divided symmetrically in male spermatogenesis. Therefore, the spermatogonial cell division may be regulated through the fusome by the diffusion of regulation factor in a direction from one end to the other end, not by the cell polarity toward original fusome or original cell.

\section{CONCLUSIONS}

In this paper, 3D structure of Drosophila testis tip was reconstructed. From this 3D structure, the size of each stage 
cell was compared in terms of volume. The stages of cells linked by the intercellular bridge in the same cyst were observed exactly and the cyst containing the same stage cells were located in the horizontal plane. The spermatogonial cells as well as the germline stem cells attached to the hub cells were divided in the almost perpendicular direction to cell layers. Furthermore, it was shown that the division in spermatogenesis may be regulated through the fusome by a different manner from the polarity in oogenesis.

\section{ACKNOWLEDGMENTS}

This work was supported by "GIST Systems Biology Infrastructure Establishment Grant (2011)" and by "the Korea Research Foundation Grant funded by the Korean Government (MOEHRD, Basic Research Promotion Fund) (KRF-2008-314-C00278)."

\section{References}

Beller, M., Riedel, D., Jansch, L., Dieterich, G., Wehland, J., Jackle, H. \& Kuhnlein, R.P. (2006). Characterization of the Drosophila lipid droplet subproteome. Mol Cell Proteomics 5, 1082-1094.

de Cuevas, M. \& Spradling, A.C. (1998). Morphogenesis of the Drosophila fusome and its implications for oocyte specification. Development 125, 2781-2789.

Deng, W. \& Lin, H. (1997). Spectrosomes and fusomes anchor mitotic spindles during asymmetric germ cell divisions and facilitate the formation of a polarized microtubule array for oocyte specification in Drosophila. Dev Biol 189, 79-94.

Fuller, M.T. (1993). Spermatogenesis. In The Development of Drosophila melanogaster, Bate, M. \& Arias, M. (Eds.), pp. 71147. New York: Cold Spring Harber Laboratory Press.
Hardy, R.W., Tokuyasu, K.T., Lindsley, D.L. \& Garavito, M. (1979). The germinal proliferation center in the testis of Drosophila melanogaster. J Ultrastruct Res 69, 180-190.

Hime, G.R., Brill, J.A. \& Fuller, M.T. (1996). Assembly of ring canals in the male germ line from structural components of the contractile ring. J Cell Sci 109, 2779-2788.

Kloc, M., Bilinski, S., Dougherty, M.T., Brey, E.M. \& Etkin, L.D. (2004). Formation, architecture and polarity of female germline cyst in Xenopus. Dev Biol 266, 43-61.

Kremer, J.R., Mastronarde, D.N. \& McIntosh, J.R. (1996). Computer visualization of three-dimensional image data using IMOD. J Struct Biol 116, 71-76.

Lighthouse, D.V., Buszczak, M. \& Spradling, A.C. (2008). New components of the Drosophila fusome suggest it plays novel roles in signaling and transport. Dev Biol 317, 59-71.

Lin, H. \& Sprading, A.C. (1995). Fusome asymmetry and oocyte determination in Drosophila. Dev Genet 16, 6-12.

Lin, H., Yue, L. \& Spradling, A.C. (1994). The Drosophila fusome, a germline-specific organelle, contains membrane skeletal proteins and functions in cyst formation. Development 120, 947-956.

Robinson, D.N. \& Cooley, L. (1996). Stable intercellular bridges in development: The cytoskeleton lining the tunnel. Trends Cell Biol 6, 474-479.

Snapp, E.L., Iida, T., Frescas, D., Lippincott-Schwartz, J. \& Lilly, M.A. (2004). The fusome mediates intercellular endoplasmic reticulum connectivity in Drosophila ovarian cysts. Mol Biol Cell 15, 4512-4521.

SPURR, A.R. (1969). A low-viscosity epoxy resin embedding medium for electron microscopy. J Ultrastruct Res 26, 31-43.

Xie, T. \& Spradling, A.C. (2000). A niche maintaining germ line stem cells in the Drosophila ovary. Science 290, 328-330.

Yamashita, Y.M., Fuller, M.T. \& Jones, D.L. (2005). Signaling in stem cell niches: Lessons from the Drosophila germline. J Cell Sci 118, 665-672. 